\title{
LA CO-PRODUCTION CONFLICTUELLE DU DROIT. LE RÔLE DES INTERMÉDIAIRES DU DROIT DANS LA JUDICIARISATION DE LA POLITIQUE SUISSE D’ASILE
}

\author{
Jonathan Miaz
}

Lextenso | «Droit et société »

2021/1 N 107 | pages 51 à 66

ISSN 0769-3362

Article disponible en ligne à l'adresse :

https://www.cairn.info/revue-droit-et-societe-2021-1-page-51.htm

Distribution électronique Cairn.info pour Lextenso.

(C) Lextenso. Tous droits réservés pour tous pays.

La reproduction ou représentation de cet article, notamment par photocopie, n'est autorisée que dans les limites des conditions générales d'utilisation du site ou, le cas échéant, des conditions générales de la licence souscrite par votre établissement. Toute autre reproduction ou représentation, en tout ou partie, sous quelque forme et de quelque manière que ce soit, est interdite sauf accord préalable et écrit de l'éditeur, en dehors des cas prévus par la législation en vigueur en France. Il est précisé que son stockage dans une base de données est également interdit. 


\title{
La co-production conflictuelle du droit. Le rôle des intermédiaires du droit dans la judiciarisation de la politique suisse d'asile
}

\author{
Jonathan Miaz
}

Centre de droit comparé européen et international (CDCEI), Université de Lausanne, Quartier UNIL-Chamberonne, Bâtiment Internef, Bureau 317, 1004 Lausanne, Suisse.

<jonathan.miaz@unil.ch>

Résumé

À partir d'une enquête sociohistorique et ethnographique, cet article analyse la judiciarisation de la politique suisse d'asile, ses ressorts et ses effets. En s'intéressant aux pratiques et aux stratégies de l'administration et de la défense juridique des migrant.e.s, il met en lumière la co-production conflictuelle du droit d'asile qui passe par des coups stratégiques et par des anticipations réciproques entre l'administration et la défense juridique, mais aussi par la production d'une jurisprudence qui participe à la sophistication du droit d'asile et de sa mise en œuvre. C'est donc d'abord au niveau de l'interprétation et de l'application du droit par l'administration que la défense juridique permet d'infléchir, à la marge, la politique d'asile.

Droit et action publique - Intermédiaires du droit - Judiciarisation Mobilisations juridiques - Politique d'asile - Street-level organizations.

Summary The Adversarial Co-Production of Law. The Role of Legal Intermediaries in the Judicialization of the Swiss Asylum Policy

This article is based on a sociohistorical and ethnographic survey. It analyzes the judicialization of the Swiss asylum policy and its practical effects. Focusing on the practices and legal strategies of the street-level bureaucracy and of the legal defense of migrants, this article argues that there is an adversarial co-production of asylum law. This process involves strategic moves and mutual anticipation between the administration and the legal defense, but also the production of case law that contributes to the sophistication of asylum law and its implementation. It is therefore primarily at the level of the interpretation and application of the law by street-level bureaucracy that legal mobilizations make it possible to change, at the margin, asylum policy.

Asylum Policy - Judicialization - Law and Policy - Legal Intermediaries Legal Mobilizations - Street-Level Organizations. 


\section{Introduction}

Durant ces dernières décennies, on observe une judiciarisation des politiques d'asile dans les pays d'Europe et d'Amérique du Nord ${ }^{1}$. Ce processus ne prend pas la même «forme » en fonction des contextes juridiques et institutionnels nationaux : les relations et les interactions entre les administrations, les avocat.e.s, les organisations actives dans la défense juridique des migrant.e.s et les tribunaux diffèrent, de même que leurs pratiques, leurs stratégies, ou l'ampleur et les effets de la jurisprudence ${ }^{2}$. Afin de saisir ce processus en pratiques et ses effets sur le terrain, cet article propose d'analyser le cas de la politique suisse d'asile non seulement d'un point de vue sociohistorique, mais aussi à partir des pratiques d'acteurs de terrain impliqués dans la chaîne d'interdépendances de la politique d'asile. Dans le cas de l'asile, ces derniers sont des intermédiaires du droit ${ }^{3}$ qui agissent en relation, souvent conflictuelle, les uns avec les autres : l'administration, à savoir le Secrétariat d'État aux migrations (SEM), les acteurs de la défense juridique des migrant.e.s, principalement des bureaux de consultation juridiques (BCJ) et des avocat.e.s privé.e.s, ainsi que le Tribunal administratif fédéral (TAF).

Cet article analyse d'abord la judiciarisation de la politique d'asile en Suisse depuis l'entrée en vigueur de la loi sur l'asile en 1981, pour s'intéresser ensuite aux pratiques des acteurs de terrain et à la manière dont les usages conflictuels du droit, respectivement de l'administration et des BCJ, configurent l'accès des requérant.e.s d'asile à une protection. En définitive, en mettant en évidence les ressorts de la judiciarisation de la politique d'asile et ses effets pratiques, cet article montre que l'on assiste à une co-production conflictuelle du droit d'asile qui passe par des coups stratégiques ${ }^{4}$ et par des anticipations réciproques entre l'administration et la défense juridique, mais aussi par la production d'une jurisprudence qui participe à la sophistication du droit d'asile et de sa mise en œuvre. C'est donc d'abord au niveau de l'interprétation et de l'application du droit par l'administration que la défense juridique permet d'infléchir, à la marge, la politique d'asile.

1. Dagmar SoEnneCKen, "The Growing Influence of the Courts over the Fate of Refugees ", Review or European and Russian Affairs, 4 (2), 2008, p. 10-43.

2. Leila KAWAR, Contesting Immigration Policy in Court: Legal Activism and Its Radiating Effects in the United States and France, New York: Cambridge University Press, coll. "Cambridge Studies in Law and Society", 2015 ; Leila KAWAR et Jonathan MIAZ, "Enacting Immigration Politics in a Juridical Register », in Shauhin TAlESH, Heinz Klug et Elizabeth MerTz (eds.), Research Handbook on Modern Legal Realism, Northampton : Edward Elgar Publishing, 2021, p. 161-175.

3. Pour S. Talesh et J. Pélisse, les intermédiaires du droit sont des acteurs étatiques, privés et de la société civile qui affectent, contrôlent ou surveillent la manière dont les règles juridiques sont interprétées, mises en œuvre ou construites une fois qu'elles sont adoptées. Shauhin TALESH et Jérôme PÉLISSE, «How Legal Intermediaries Facilitate or Inhibit Social Change », Studies in Law, Politics and Society, 79, 2019, p. 111-145.

4. Cette notion, en référence à celle « d'échange de coups » utilisée en sociologie des crises politiques, vise à saisir l'activité tactique des acteurs et les anticipations réciproques des pratiques des autres acteurs : Hervé RAYNER, "Quelques apports de Thomas Schelling à la compréhension des processus sociaux ", Émulations - Revue de sciences sociales, 31, 15 novembre 2019, p. 105-115. 


\section{Méthodes et terrains d'enquête}

Cet article repose sur une enquête ethnographique visant à analyser la politique suisse d'asile à partir des pratiques du SEM, des BCJ et du TAF 5 . J'ai d'abord conduit une recherche sociohistorique à partir de diverses sources (archives fédérales en ligne, jurisprudence, etc.) et en constituant un dossier de presse depuis les années 1970. De 2010 à 2012, j'ai effectué deux enquêtes de terrain de plusieurs mois au sein d'un Centre d'enregistrement et de procédure (CEP) où les migrant.e.s pouvaient déposer leur demande d'asile, puis au sein de la division « Procédure à la centrale et retour » du SEM à Berne. En tant qu'observateur externe, j'ai assisté à des auditions d'asile, suivi des formations internes et mené 59 entretiens semi-directifs avec des agent.e.s chargé.e.s d'instruire les demandes d'asile (ci-après «spécialistes asile») ainsi qu'avec des cadres intermédiaires du SEM. Ces stages d'observation m'ont permis de consulter divers documents de travail du SEM. En 2011, j'ai également effectué un stage d'observation participante de six mois en tant que bénévole au sein d'un BCJ, complété par vingt entretiens semi-directifs en 2012 et 2013 avec des juristes et des bénévoles de plusieurs BCJ situés dans différents cantons suisses, ainsi qu'avec quelques avocat·e.s spécialisée.e.s. En 2018 et 2019, j'ai conduit quinze entretiens semi-directifs supplémentaires au sein de plusieurs BCJ. Enfin, j'ai mené des entretiens avec trois juges et trois greffiers.ères du Tribunal administratif fédéral en 2013.

\section{Des street-level organizations interdépendantes : une analyse relationnelle de la mise en œuvre d'une politique d'asile}

Cet article analyse la judiciarisation de la politique suisse d'asile en pratique et la production relationnelle du droit. La judiciarisation est une notion polysémique qui désigne ici le processus par lequel la justice devient une actrice importante du politique et de l'action publique ${ }^{6}$. Ainsi, au-delà de leur hétérogénéité, les travaux qui portent sur ce processus permettent de saisir les interdépendances croissantes entre les champs politique et judiciaire dans la fabrication de l'action publique. Ces dynamiques doivent donc être saisies comme étant un effet de «l'allongement progressif des chaînes d'interdépendance dont procèdent les politiques publiques qui incluent désormais une diversité plus grande de spécialistes et “d'experts", parmi lesquels peuvent prendre place des juristes et des juges ${ }^{7}$. Partant, nous adoptons une approche relationnelle ${ }^{8}$ de la mise en œuvre de la politique d'asile et de sa judi-

5. Cet article ne porte pas sur la nouvelle procédure d'asile entrée en vigueur le $1^{\text {er }}$ mars 2019 et qui introduit une représentation juridique gratuite.

6. Pour des revues de littérature, voir : Jacques COMMAILlE et Laurence DuMOULIN, « Heurs et malheurs de la légalité dans les sociétés contemporaines. Une sociologie politique de la "judiciarisation" ", L'Année sociologique, 59 (1), 2009, p. 63-107 ; Rebecca HAMLIN et Gemma SALA, "The Judicialization of Politics Disentangled ", in William R. THOMPSON (ed.), Oxford Research Encyclopedia of Politics, New York: Oxford University Press, 2018, vol. 1, p. 1-29.

7. Laurence Dumoulin et Violaine Roussel, « La judiciarisation de l'action publique », in Olivier BorRAz et Virginie Guiraudon (dir.), Politiques publiques 2, Changer la société, Paris: Presses de Science Po, 2010, p. 253.

8. Ibid. ; Leila KAWAR, Contesting Immigration Policy in Court: Legal Activism and Its Radiating Effects in the United States and France, op. cit. ; Hervé RAYNER et Bernard VOUTAT, «La judiciarisation à l'épreuve de la démocratie directe. L'interdiction de construire des minarets en Suisse », Revue française de science politique, 64 (4), 2014, p. 689-709 ; Alec STONE SwEET, «Judicialization and the Construction of Governance », Comparative Political Studies, 32 (2), 1999, p. 147-184. 
ciarisation en nous concentrant sur les pratiques des acteurs de terrain, mettant en évidence les ressorts et les effets d'un certain recours à l'institution judiciaire sur l'application du droit d'asile.

Cette perspective s'intéresse aux relations entre les organisations impliquées dans cette chaîne d'interdépendances. Il s'agit d'étudier en particulier les rapports entre l'État et les organisations de défense des droits des migrant.e.s pour saisir comment leurs pratiques et leurs stratégies interagissent, se répondent et s'influencent réciproquement ${ }^{9}$ pour produire in fine le droit d'asile au concret. Autrement dit, cet article analyse la mise en œuvre du droit à partir des pratiques de différents acteurs : non seulement de l'administration et du tribunal, mais aussi des avocat.e.s et des BCJ 10 qui offrent un accès à une protection juridique, et donc un accès au(x) droit(s) ${ }^{11}$, aux requérant.e.s d'asile en transformant leurs demandes en actions "constituées juridiquement ", c'est-à-dire formulées de manière "audible » dans le cadre de la saisine du tribunal administratif 12 .

Ces différents acteurs sont considérés comme des intermédiaires du droit qui se situent entre les règles juridiques formelles et les personnes qui sont concernées par la politique d'asile ${ }^{13}$. Cette approche permet ainsi de mettre en évidence comment ils appliquent et mobilisent le droit, en travaillent (ou contestent) les catégories juridiques et en (re)définissent les contours non seulement via les conséquences pratiques des décisions administratives, des recours déposés par les avocat·e·s et les BCJ, ainsi que de la jurisprudence du tribunal, mais aussi à travers les effets de l'ensemble de ces pratiques sur les révisions législatives. Autrement dit, une analyse en termes d'intermédiation juridique appliquée à ces différents acteurs s'intéresse à la manière dont ils font usage des règles juridiques, ce qui permet de comprendre si et comment ils influencent le droit en action et la production du droit 14 .

De manière complémentaire, ces acteurs sont aussi considérés comme des streetlevel organizations (SLO) 15 impliquées dans la mise en œuvre et la production in

9. Sur cet aspect, voir : Cary COGLIANESE, «Litigating within Relationships: Disputes and Disturbance in the Regulatory Process », Law \& Society Review, 30 (4), 1996, p. 735-766 ; Aude LejEunE, "Legal Mobilization within the Bureaucracy: Disability Rights and the Implementation of Antidiscrimination Law in Sweden ", Law \& Policy, 39 (3), 2017, p. 237-258. ; Anne ReviLLARD, « Social Movements and the Politics of Bureaucratic Rights Enforcement: Insights from the Allocation of Disability Rights in France ", Law \& Social Inquiry, 42 (2), 2017, p. 450-478.

10. Leila KAWAR, «Legal Mobilization on the Terrain of the State: Creating a Field of Immigrant Rights Lawyering in France and the United States », Law \& Social Inquiry, 36 (2), 2011, p. 354-387.

11. Dominique Belkis, Spyros FranguIAdiakis et Edith JAILLARDOn, En quête d'asile. Aide associative et accès $a u(x) \operatorname{droit}(s)$, Paris : LGDJ, 2004.

12. Jean-Gabriel CONTAMIn, Emmanuelle SAADA, Alexis SPIRE et Katia WeIDENFELD, Le recours à la justice administrative. Pratiques des usagers et usages des institutions, Paris : La Documentation française, 2008.

13. Jérôme PÉLISSE, «Varieties of Legal Intermediaries: When Non-Legal Professionals Act as Legal Intermediaries ", Studies in Law, Politics and Society, 81, 2019, p. 101-128.

14. Ibid., p. 104.

15. Dans la continuité de l'ouvrage fondateur de Michael Lipsky sur la Street-level bureaucracy, la notion de SLO permet d'inclure dans l'analyse d'autres organisations (non-gouvernementales, associatives, privées, à but lucratif ou non) que les administrations publiques, qui sont aussi impliquées dans la mise en œuvre des politiques publiques. Voir : Evelyn Z. BRODKIN et Pierre-Yves BAUdOT, «Les agents de terrain, entre politique et action publique », Sociologies pratiques, 24 (1), 2012, p. 10-18. 
concreto de l'action publique ${ }^{16}$. Dans cette perspective, les SLO sont des lieux où se poursuit la lutte politique pour la définition de l'action publique ${ }^{17}$. Plusieurs recherches ont ainsi analysé la manière dont les administrations instruisent les demandes d'asile en accordant une place importante à la question de la crédibilité des récits, plaçant le soupçon au cœur de la procédure d'asile ${ }^{18}$, et comment leurs agent.e.s construisent et appliquent des catégories de traitement qui façonnent la catégorie de réfugié.e ${ }^{19}$. D'autres travaux ont mis en évidence les tensions au sein des associations en lien avec leur implication dans la mise en œuvre des politiques d'asile ou, du moins, des catégories juridico-administratives ${ }^{20}$. Il s'agit donc ici d'analyser la manière dont le conflit autour de la politique d'asile et de ses contours se poursuit en pratique dans les usages conflictuels du droit de ces différents acteurs de terrain. En définitive, cette approche cherche à saisir le "processus de création normatif continu » 21 et relationnel, c'est-à-dire comment le droit d'asile est co-produit par les interactions entre les pratiques et usages (conflictuels) du droit de différents intermédiaires impliqués dans la phase de mise en œuvre de la politique d'asile.

\section{La judiciarisation de la politique suisse d'asile}

La première loi sur l'asile (LAsi) est entrée en vigueur en 1981. Durant les années 1980, l'asile est rapidement devenu l'une des thématiques les plus débattues de l'agenda politique suisse ${ }^{22}$. Au cours des quatre dernières décennies, on observe une inflation normative, la loi sur l'asile étant modifiée à de nombreuses reprises au nom de "la lutte contre les abus" et de «l'accélération des procédures ». Cette évolution se caractérise par de nombreux durcissements de la loi : restrictions de l'accès au marché de l'emploi, aux prestations sociales ou à la procédure d'asile, resserrement de l'interprétation de la notion de réfugié et renforcement des exi-

16. Vincent DuBOIS, « Politiques au guichet, politiques du guichet », in Olivier BorRAz et Virginie GuIRAUDON (dir.), Politiques publiques 2, Changer la société, op. cit., p. 265-286.

17. Sur les “SLOs as Sites of Policy Conflict," voir : Evelyn Z. BRODKIN, « Street-Level Organizations and the Welfare State", in Evelyn Z. BRodKIn et Gregory MARSTON (eds.), Work and the Welfare State. Street-Level Organizations and Workfare Politics, Washington, DC : Georgetown University Press, 2013, p. 17-34.

18. Voir par exemple : Olga JuBANY, Screening Asylum in a Culture of Disbelief: Truths, Denials and Skeptical Borders, Cham : Palgrave Macmillan 2017; Johanna PRoBST, «Entre faits et fiction : l'instruction de la demande d'asile en Allemagne et en France », Cultures \& Conflits, 84, 2011, p. 63-80.

19. Karen АкоКА, «La fabrique des réfugiés dans la Guerre froide : une ethnographie historique des "vingt glorieuses" de l'attribution de l'asile en France (1952-1972) ", Politique et Sociétés, 38 (1), 2019, p. 19-48.

20. Éric Agrikoliansky, «Usages choisis du droit: le service juridique de la ligue des droits de l'homme (1970-1990). Entre politique et raisons humanitaire », Sociétés contemporaines, 52 (4), 2003, p. 61-84 ; Estelle D'HALluin, «Passeurs d'histoire. L'inconfort des acteurs associatifs impliqués dans l'aide à la procédure d'asile", in Didier FASsin (dir.), Les nouvelles frontières de la société française, Paris : La Découverte, 2010, p. 363-383 ; Mathilde PETTE, «Associations : les nouveaux guichets de l'immigration ? Du travail militant en préfecture», Sociologie, 5 (4), 2014, p. 405-421.

21. Pierre LASCOUMES, « Normes juridiques et mise en œuvre des politiques publiques », L'Année sociologique, 40, 1990, p. 43-71.

22. Lorena PARINI et Matteo GIANNI, « Enjeux et modifications de la politique d'asile en Suisse de 1956 à nos jours ", in Hans MAHNIG (dir.), Histoire de la politique de migration, d'asile et d'intégration en Suisse depuis 1948, Zurich : Éditions Seismo, 2005, p. 189-252. 
gences en termes de crédibilité des récits. Toutes ces révisions ont été adoptées par le Parlement et sept d'entre elles ont été acceptées par le peuple après un référendum, conférant aux durcissements de la politique d'asile une certaine légitimité populaire.

\section{II.1. Vers la création d'une instance de recours indépendante de l'administration}

Durant les années 1980, la création d'une autorité de recours indépendante de l'administration était l'une des revendications du mouvement de défense des migrant·e.s 23 qui contestait principalement "l'illégalité » et "l'arbitraire » des pratiques de l'État à une époque où le service de recours interne au Département fédéral de justice et police (DFJP) confirmait les décisions des autorités dans 99,96\% des cas ${ }^{24}$. À la fin des années 1980 , il n'existait en effet que cette voie de recours interne de l'administration 25. À plusieurs reprises, les demandes de création d'une instance indépendante sont rejetées par le Conseil fédéral et par le Parlement. L'enjeu était de préserver le contrôle sur la pratique en évitant de se soumettre à une juridiction administrative susceptible de réduire la marge de manœuvre des autorités dans la conduite de la politique d'asile. Le Conseil fédéral explique ainsi : « une telle commission représenterait une juridiction administrative susceptible d'orienter sensiblement la pratique de l'asile, sans en référer aux autorités politiques » et « le droit du Conseil fédéral d'édicter des directives ne serait pas préservé non plus en la circonstance de sorte que la plus haute autorité politique serait privée de la possibilité d'intervenir dans la jurisprudence en matière de recours » 26 . C'est aussi pour cette raison et parce que l'asile n'est pas reconnu comme un droit subjectif, que les recours au Tribunal fédéral (plus haute juridiction suisse) sont exclus.

Les autorités fédérales vont cependant revenir sur leur position en 1990. La création d'une instance de recours indépendante vise alors à alléger la charge de travail du DFJP et à augmenter la légitimité des décisions d'octroi de l'asile ou de renvoi, «ce qui devrait avoir pour effet de diminuer le recours à ces moyens et ces voies de droit extraordinaires " 27 . La Commission de recours en matière d'asile (CRA) entre en fonction le $1^{\text {er }}$ avril 1992 et sera remplacée en 2007 par les cours IV et V du Tribunal administratif fédéral (TAF) lors de la réforme de l'organisation judiciaire suisse. Initialement, l'administration, le Parlement et le Conseil fédéral étaient réticents à la mise en place d'une telle instance dont la création est qualifiée « d'acte

23. Jonathan MiAz, Politique d'asile et sophistication du droit. Pratiques administratives et défense juridique des migrants en Suisse (1981-2015), thèse de doctorat en science politique, Université de Lausanne et Université de Strasbourg, 2017.

24. Jonathan PäRLI, « Legal, illegal... - wer genau? Die Schweizer Asylbewegung und das Recht 1973-1992 ", Zeitschrift für Rechtssoziologie, 39 (2), 2019, p. 177-203.

25. Il existait auparavant une deuxième voie de recours auprès du Conseil fédéral (gouvernement), supprimée lors de la première révision de la LAsi entrée en vigueur en 1984.

26. Message sur la révision de la loi sur l'asile du 6 juillet 1983, 83.046, FF 1983 III 38, p. 819-820.

27. Message à l'appui d'un arrêté fédéral sur la procédure d'asile (APA) et d'une loi fédérale instituant un Office fédéral pour les réfugiés du 25 avril 1990, FF 1990 II, p. 540. 
politique ${ }^{28}$. Pour eux, les décisions rendues en matière d'asile sont de « nature politique » 29 et la création d'une instance de recours indépendante leur fait craindre de perdre le contrôle sur l'application du droit d'asile, ce qui réduirait leur pouvoir discrétionnaire en la matière.

Du côté des mouvements de défense des migrant·e·s, dès les années 1980 et plus encore dans les années 1990, on observe une juridicisation des modalités d'action de nombreuses organisations dans le sens où elles se tournent largement vers la défense juridique individuelle en créant des bureaux de consultation juridique spécialisés dans le domaine de l'asile et en recrutant des juristes ${ }^{30}$. Ces BCJ sont les principaux acteurs auprès desquels les requérant.e.s d'asile peuvent solliciter une aide juridique, généralement gratuite.

\section{II.2. Dimensions quantitatives et qualitatives de la judiciarisation}

On peut parler de judiciarisation de la politique d'asile à un double titre. Quantitativement d'abord, l'asile est l'un des domaines du droit administratif qui est le plus « judiciarisé » au niveau fédéral, en termes de nombre de recours déposés 31 , ce qui témoigne de la dimension conflictuelle de ce droit, imputable à des usages spécifiques du recours et une défense juridique essentiellement individuelle. Cette judiciarisation ne se présente pas comme une augmentation progressive du volume des recours. En effet, ce dernier fluctue, notamment en fonction du nombre de décisions rendues par l'administration. Il est aussi lié aux ressources limitées des migrant·e.s pour recourir à un·e avocat.e, ainsi que des BCJ qui sélectionnent les dossiers qu'ils peuvent défendre ${ }^{32}$. Le graphique ci-dessous présente l'activité de l'instance de recours en matière d'asile (nombre d'affaires réglées) en parallèle des décisions rendues par l'administration sur les demandes d'asile. Il montre que la judiciarisation de la politique suisse d'asile consiste en un volume important et permanent d'arrêts rendus sur des recours déposés contre des décisions de l'administration. Ce nombre d'affaires réglées par le TAF s'est stabilisé autour de la barre des 5000 par année et varie assez peu depuis 2007.

28. Déclaration personnelle de M. Jeanneret durant le débat parlementaire du 5 juin 1990 sur « Procédure d'asile. Modification» (90.025). Référence : BO CN 1990, p. 819.

29. Office fédéral des migrations, «\$1 La protection juridique dans la procédure d'asile», in Manuel de Procédure d'asile, consulté en 2009 sur l'ancien site web de l'ODM : <http://bfm.admin.ch>. La version en ligne actuelle a été actualisée et modifiée.

30. Cette dynamique s'est encore renforcée avec la restructuration du domaine de l'asile entrée en vigueur en 2019. Cette réforme a introduit une assistance juridique gratuite dans les centres fédéraux où se déroule la procédure. Ainsi, le SEM a attribué des mandats de prestation à plusieurs organisations déjà actives dans la défense juridique. Sur cette réforme et ses effets sur les organisations de défense des migrant·e.s, voir : Tobias EulE, "Advice as a Vocation? Politics, Managerialism and State Funding in the Swiss Refugee Support Community", Ethnos, 2020, p. 1-16.

31. Thierry TANQUerel, Frédéric Varone, Arun BolKensteyn et Karin Byland, Le contentieux administratif en Suisse : une analyse empirique, Genève : Schulthess, 2011.

32. Jonathan MIAZ, «Qui peut rester et qui doit partir ? Les frontières au prisme des usages sociaux du droit d'asile en Suisse ", Trajectoires [En ligne], Hors série 3, 2017, <https://journals.openedition.org/trajectoires/2398>. 


\section{Décisions de l'administration et affaires réglées par l'instance de recours (CRA, TAF) en matière d'asile 33}

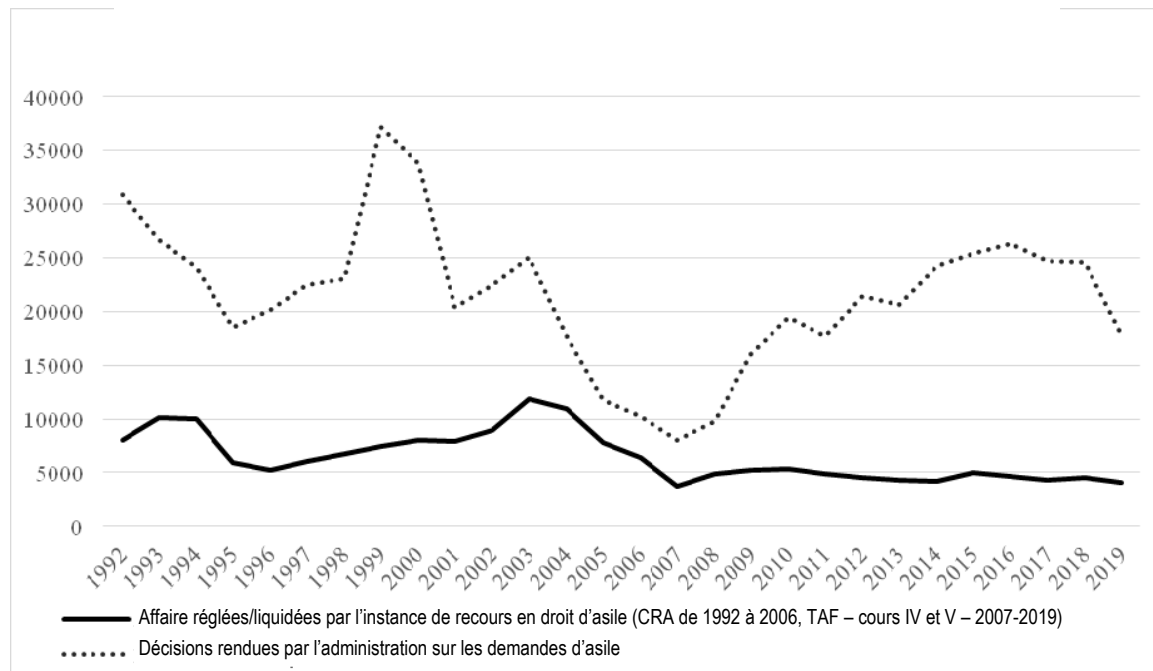

Depuis 1992, les juges ont développé une jurisprudence dans un domaine qui en était presque vierge ${ }^{34}$. Les arrêts étant de plus en plus approfondis, elle est devenue plus complexe, précise et nuancée. Ainsi, c'est aussi qualitativement que l'on peut parler de judiciarisation de la politique d'asile. En effet, depuis 1992, la jurisprudence a précisé différents aspects du droit d'asile, en apportant des détails et des réponses pratiques 35 qui orientent les usages bureaucratiques et contestataires du droit. Elle a concerné des questions substantielles, telles que la définition du réfugié et la notion de persécution, aussi bien que procédurales, liées par exemple au droit de la preuve et à la notion de vraisemblance.

Finalement, cette jurisprudence a considérablement spécifié l'interprétation et l'application du droit d'asile en fonction des différents motifs et situations des requérant.e.s d'asile, analysés en fonction du contexte des pays d'origine. Cela a conduit à une sophistication du droit, c'est-à-dire à une évolution de l'encadrement juridique dans le sens d'une plus grande complexité, impliquant une diversification des niveaux normatifs, un niveau d'élaboration (technique) plus poussé et une spécification croissante des règles de droit en fonction des situations particulières des requérant.e.s d'asile et de leurs pays d'origine. Ce processus a des effets importants sur les agent.e.s du SEM qui doivent conformer leurs pratiques et leurs normes secondaires d'application à la jurisprudence, et sur la défense juridique qui

33. Ce graphique combine les données concernant les affaires réglées par les cours IV et V du TAF que j'ai compilées à partir de ses rapports de gestion annuels de 2007 à 2019, avec les données récoltées par Thierry TANQUEREL et al., Le contentieux administratif en Suisse: une analyse empirique, op. cit. dans le cadre du projet FNRS 100015-118022. Je remercie Karin Byland de m'avoir communiqué ces données.

34. Entretien au TAF, 2013.

35. On observe tout particulièrement cela dans les manuels et les recueils annuels de jurisprudence. 
peut utiliser des «brèches » ou des jurisprudences «favorables » pour défendre les migrant·e.s, mais qui doit aussi faire avec des arrêts plus restrictifs entérinant des durcissements de pratique.

\section{Une défense juridique entre aide individuelle, contestation des pratiques administratives et tentatives de changer la jurisprudence}

Les opportunités juridiques pour contester la pratique du SEM en matière d'asile sont limitées. En termes de contrôle judiciaire de la mise en œuvre du droit d'asile, il n'existe qu'une voie de recours (TAF) qui statue sur des cas individuels, présentés uniquement sous forme écrite (sans audience) avec de possibles frais de procédure. Il n'y a pas de contrôle de constitutionnalité des lois fédérales ${ }^{36}$ et il n'existe pas de mécanisme de class action ou de recours collectifs comme aux ÉtatsUnis, ni d'instance supérieure, comme le Conseil d'État en France, auprès de qui contester des circulaires ou des directives administratives ${ }^{37}$. Cette partie analyse les pratiques et les stratégies juridiques des BCJ pour défendre les droits des migrant·e.s, pour contester ou infléchir les modalités de la mise en œuvre du droit d'asile et, à la marge, pour le transformer via la jurisprudence.

Même si l'on observe différents types de rapports au rôle et à la cause de l'asile, la plupart des juristes et des bénévoles des BCJ considèrent qu'ils et elles défendent «la cause de l'asile» 38 , voire « le droit des gens de rester ici, ou le droit d'avoir la chance de s'intégrer dans une société qui offre des perspectives » 39 . Ainsi, défendre les requérant.e.s d'asile à travers des recours individuels apparaît comme une manière de revendiquer, voire de militer pour une «bonne application» du droit d'asile. Dans cette perspective l'usage du droit vise à faire valoir les droits des personnes, à faire correspondre l'application du droit objectif (law) avec les droits subjectifs (rights) des individus 40 . L'action des BCJ consiste principalement à fournir une aide juridique gratuite aux requérant.e.s d'asile en les conseillant juridiquement et, en fonction des cas, en déposant des recours individuels ou en rédigeant des demandes de reconsidération de la demande d'asile. Les conseiller.ère-s juridiques sont souvent des juristes, même si l'on trouve également des personnes disposant d'un diplôme en sciences sociales (anthropologie, sociologie, science politique, etc.), et peuvent

36. Selon l'article 189, al. 4 de la Constitution suisse, «les actes de l'Assemblée fédérale et du Conseil fédéral ne peuvent pas être portés devant le Tribunal fédéral », sauf si une loi le prévoit explicitement ou si une protection judiciaire découle du droit international.

37. Éric AgRIKOLIANSKY, «Usages choisis du droit: le service juridique de la ligue des droits de l'homme (1970-1990). Entre politique et raisons humanitaire ", article cité ; Liora IsRAËL, "Faire émerger le droit des étrangers en le contestant, ou l'histoire paradoxale des premières années du GISTI ", Politix, 62, 2003, p. 115-143; Leila KAWAR, Contesting Immigration Policy in Court : Legal Activism and Its Radiating Effects in the United States and France, op. cit.

38. Entretien avec J., bénévole au BCJ-VS, mai 2013. Pour des raisons de confidentialité, les prénoms et initiales des personnes citées ont été changés.

39. Entretien avec M., juriste au BCJ-VD, juin 2013.

40. Liora ISRAËL, «Qu'est-ce qu'avoir le droit ? Des mobilisations du droit en perspective sociologique », $L e$ sujet dans la Cité. Revue internationale de recherche biographique, 3, 2012, p. 34-47. 
être considérés comme des cause lawyers ${ }^{41}$ qui s'engagent pour la cause des migrant.e.s, dans leur pratique professionnelle.

Les objectifs poursuivis par les BCJ sont principalement d'informer, d'orienter, de représenter et de défendre juridiquement les personnes migrantes. Durant les permanences, les conseiller-ère-s juridiques reçoivent les personnes individuellement et doivent décider s'ils et elles acceptent de prendre un mandat de défense juridique. En effet, en raison de ressources (financières, en temps et en personnel) insuffisantes pour absorber les nombreuses demandes d'aide qui leur sont adressées durant les permanences, les BCJ sont contraints de "sélectionner» celles et ceux qu'ils vont prendre en charge pour pouvoir « les faire à fond " 42 .

La sélection repose principalement sur une évaluation des « chances de succès " d'une requête auprès des autorités (SEM, TAF, Cour européenne des droits de l'homme - CEDH, Comité contre la torture - CAT), c'est-à-dire des possibles juridiques que les juristes des BCJ peuvent estimer en fonction de leur connaissance des pratiques administratives et judiciaires correspondant aux situations particulières qui leur sont soumises. Cette évaluation conduit à reprendre, dans une certaine mesure, les critères juridico-administratifs des autorités. Elle repose aussi sur les "prises » qu'offrent non seulement le droit et la jurisprudence, mais aussi les dossiers eux-mêmes et d'éventuelles expertises médicales ou sur les pays d'origine permettant «d'avoir quelque chose à dire » 43 , c'est-à-dire d'étayer une argumentation dans le cadre d'un recours ou d'une demande de reconsidération. Ainsi, pour évaluer si un dossier est "voué à l'échec" ou non, "il faut connaître la jurisprudence en la matière " et être en mesure de "contrer tous les arguments du SEM, [...] ou la majorité, sur des points précis » 44 , à la fois concernant la vraisemblance, la pertinence et l'évaluation de l'exigibilité ou de la licéité du renvoi dans le pays d'origine ou dans un autre État ayant adopté le règlement de Dublin.

À côté de ce critère de sélection, les juristes et les bénévoles des BCJ se basent également sur des considérations morales et politiques ${ }^{45}$. La défense individuelle peut donc aussi être une manière de contester la politique poursuivie par les autorités, en mobilisant le registre des droits humains et du droit humanitaire, en particulier lorsqu'ils et elles estiment qu'il y a une violation des droits fondamentaux par les autorités. Ce registre conduit souvent à passer outre l'absence de chances de succès pour tout de même faire recours et manifester un désaccord «avec la politique du Gouvernement » 46 . Une autre juriste m'expliquait qu'elle prenait systématiquement un mandat pour les Roms des Balkans pour dire aux autorités :

41. Liora ISRAËL, «Usages militants du droit dans l'arène judiciaire : le cause lawyering », Droit et Société, 49, 2001, p. 793-824.

42. Entretien avec V., juriste au BCJ-NE, mai 2013.

43. Entretien avec S., juriste au BCJ-VD, juin 2013.

44. Entretien avec N., juriste au BCJ-FR, août 2018.

45. Voir: Camille HAMIDI, "Associations, politisation et action publique. Un monde en tensions", in Olivier Fillieule, Florence HaEgel, Camille Hamidi et Vincent Tiberj (dir.), Sociologie plurielle des comportements politiques. Je vote, tu contestes, elle cherche..., Paris : Presses de Sciences Po, 2017, p. 360.

46. Entretien avec D., juriste au BCJ-VD, juin 2013. 
Je ne suis absolument pas d'accord avec ce que vous faites. [...] Nous, on estime que les Roms sont discriminés, c'est un critère de la loi sur l'asile. Donc pour nous, les Roms ne doivent pas être renvoyés 47 .

L'action des BCJ et des juristes est limitée par les dispositifs juridiques et administratifs de la procédure d'asile et de renvoi ${ }^{48}$ qui pose un certain nombre de contraintes concernant la forme et les arguments qui peuvent être invoqués et qui n'offre que des opportunités limitées pour contester la politique et les pratiques des autorités ${ }^{49}$. Cela peut générer une forme de frustration :

Je suis un peu énervée parce que je sais que mon travail se limite à faire des petits trucs sans pouvoir changer quelque chose qui, à la base déjà, n'est pas bien. [...] Moi, je pense que le droit, c'est comme un outil, mais qu'il est très limité. Parce que moi, je ne peux pas inventer. Je ne peux que prendre ce qui est déjà là dans la loi ou dans les décisions du TAF, dans la jurisprudence, mais je ne peux pas aller au-delà 50 .

Si l'usage du droit reste limité aux prises qu'il offre, plusieurs juristes soulignent qu'ils et elles luttent surtout contre une certaine application du droit, contre la manière dont l'administration utilise sa marge d'appréciation et interprète les situations et les risques des personnes ${ }^{51}$. En effet, pour plusieurs juristes :

Ce n'est pas très important ce qui est écrit dans la loi, c'est la manière dont les gens, dont les autorités l'appliquent. C'est ça qui nous ferme les portes 52 .

Ainsi, l'usage du droit défendu par les BCJ ne vise pas à désobéir au droit 53, à le subvertir ou à utiliser la justice comme une arène politique. Il s'agit plutôt d'agir sur sa mise en œuvre, c'est-à-dire de défendre une autre interprétation et une autre application du droit d'asile et des droits fondamentaux. En ce sens, les BCJ sont des intermédiaires du droit qui participent à la mise en œuvre conflictuelle du droit d'asile et des droits fondamentaux.

Toutefois, au-delà des cas individuels, les juristes expriment aussi une volonté de modifier le droit à travers la jurisprudence :

Dans des situations très rares, on prenait aussi le mandat quand on voyait qu'il y avait une jurisprudence bien précise en la matière, mais qu'on avait de nouveaux arguments, qu'on aimerait bien pousser la jurisprudence, et peut-être qu'elle change sur certains aspects. Ça, c'était carrément le cas quand on a commencé à faire recours pour des cas Dublin Hongrie. Au début, c'était voué à l'échec, mais après, on a eu de nouveaux éléments [...]. J'avais des informations des ONG hongroises et aussi d'autres éléments qui ont fait qu'on a décidé de faire un peu de la stratégie 54 .

47. Entretien avec J., bénévole au BCJ-VS, mai 2013.

48. Raphaël REY et Olivier BEURRET, « "Humainement je vous comprends; juridiquement, je ne peux rien faire" : une analyse du conseil juridique associatif dans la procédure d'asile suisse ", in Marion FrESIA, David BozZINI et Alice SALA (dir.), Les rouages de l'asile en Suisse. Regards ethnographiques sur une procédure administrative, Neuchâtel : SFM, collection «Études du SFM », 62, 2013, p. 63-92.

49. Liora IsRaËL, L'arme du droit, Paris : Presses de Sciences Po, 2009 ( $2^{\mathrm{e}}$ éd. parue en 2020).

50. Entretien avec L., juriste au BCJ-BS, août 2018.

51. Entretien avec L., juriste au BCJ-VD, août 2018.

52. Entretien avec M., juriste au BCJ-VD, juin 2013.

53. Mauricio GARCíA VILLEGAS et Aude LEJEUNE, «La désobéissance au droit : approche sociologique comparée », Droit et Société, 91, p. 565-577.

54. Entretien avec M., juriste au BCJ-FR, août 2018. 
Selon cette juriste, d'autres BCJ ont déposé de nombreux recours basés sur cette argumentation. Le TAF a alors suspendu les décisions de renvoi Dublin vers la Hongrie jusqu'à ce que la CEDH rende un arrêt de référence obligeant le SEM à évaluer s'il y a des défaillances systémiques en Hongrie. On voit ainsi l'importance prise par les expertises sur les pays d'origine (country of origin information-COI 55 ).

Ainsi, d'une part, les BCJ se concentrent sur la défense individuelle et configurent un accès $\mathrm{au}(\mathrm{x})$ droit(s), c'est-à-dire à un conseil et une défense permettant d'agir par le droit 56 contre une décision individuelle pour faire valoir ses droits et obtenir l'asile ou une admission provisoire. À travers le nombre important de recours déposés contre les décisions du SEM, cette défense individuelle conteste massivement les pratiques administratives et constitue une forme de contre-pouvoir et de contrôle de l'administration. Les BCJ s'opposent à la mise en œuvre de la politique d'asile par l'administration en procédant à ce que l'on peut qualifier de "guérilla juridique $" 57$ - soit une contestation juridique répétée, continue et quasi systématique des décisions administratives - qui vise à infléchir les pratiques de l'administration travaillant sous la «menace " permanente des recours. Dans la continuité de Evelyn Z. Brodkin ${ }^{58}$, on peut dire que les BCJ poursuivent le conflit autour de la politique d'asile sur le terrain de l'application du droit d'asile afin d'en défendre une interprétation moins restrictive et plus respectueuse des droits fondamentaux. Leur marge de manœuvre reste toutefois largement conditionnée par les prises qu'offrent le droit, les dossiers et les expertises à disposition des BCJ, mais aussi par leurs interactions avec les autorités (SEM, TAF) et par les pratiques de ces dernières, ce qui souligne encore la dimension relationnelle du travail d'intermédiation juridique.

En outre, depuis quelques années, on observe une organisation croissante des acteurs de la défense juridique, notamment autour de réunions de coordination entre BCJ (Copera, RBS treffen) et de l'Organisation suisse d'aide aux réfugiés (OSAR), pour développer des stratégies visant à changer certaines jurisprudences. En 2014, le Centre suisse pour la défense des droits des migrants (CSDM) a été créé pour se concentrer sur des litiges stratégiques 59 auprès d'instances internationales telles que la CEDH ou le Comité de l'ONU contre la torture ${ }^{60}$. Les stratégies des BCJ et du CSDM visent à transformer le droit et son application par les autorités, c'est-à-dire à

55. Damian Rosset, « Le savoir sur les pays d'origine dans les procédures d'asile. Construction et négociation institutionnelle de la réalité ", Jusletter, 16 mars 2015.

56. Jérôme Drahy, Le droit contre l'État ? Droit et défense associative des étrangers : l'exemple de la CIMADE, Paris : L'Harmattan, 2004.

57. À propos du GISTI, en France, Liora Israël parle de « guérilla judiciaire » (Liora ISRAËL, « Faire émerger le droit des étrangers en le contestant, ou l'histoire paradoxale des premières années du GISTI ", op. cit., p. 141). La notion de "guérilla juridique" se veut plus large pour inclure le recours à d'autres démarches ou stratégies juridiques telles que la procédure de réexamen de la demande d'asile (Jonathan MiAz, Politique d'asile et sophistication du droit, op. cit.).

58. Evelyn Z. BRodKIN, « Street-Level Organizations and the Welfare State », op. cit.

59. Aude LejEune et Jean-François ORIANNE, « Choisir des cas exemplaires : la strategic litigation face aux discriminations ", Déviance et Société, 38 (1), 2014, p. 55-76.

60. Entretien avec le directeur du CSDM, novembre 2019. 
agir sur le droit ${ }^{61}$. Ce faisant, ces intermédiaires du droit favorisent un changement juridique 62 " par la marge », via des jurisprudences favorables.

\section{L'administration face à sa contestation : anticipations et jeux stratégiques}

La proportion élevée de recours déposés contre les décisions de l'administration amplifie la dimension conflictuelle des relations entre acteurs de terrain au niveau de la mise en œuvre du droit. Cette conflictualité a des effets ambivalents sur les pratiques des "spécialistes asile" du SEM. Premièrement, la "menace du recours " peut dans le même temps renforcer la rigueur juridique dont font preuve les agent·e·s qui cherchent à "bétonner" leur décision, mais elle peut aussi être considérée comme un "filet de sécurité" permettant de corriger d'éventuelles erreurs, et de "sauver" celles et ceux à qui une protection aurait dû être accordée 63 . Ainsi, si, d'un côté, des « spécialistes asile " reconnaissent que le rôle des BCJ est nécessaire, de l'autre, plusieurs agent.e.s regrettent et critiquent les pratiques conflictuelles des BCJ 64 . Certain.e.s considèrent aussi que ces BCJ défendent souvent des « causes perdues » ou des " cas indéfendables », afin de s'opposer systématiquement à l'État ou pour défendre l'idée que tout le monde a le droit de rester en Suisse, ce qu'ils et elles estiment comme étant « irresponsable»65, d'autant que les recours et les demandes de reconsidération tendent à augmenter leur charge de travail.

Au-delà des pratiques individuelles des «spécialistes asile », cette forme de judiciarisation avec une jurisprudence importante et en constante évolution a des effets plus larges sur lesdites «pratiques d'asile » élaborées par les «spécialistes asile» et les cadres intermédiaires du SEM. Pour chaque pays d'origine, ces normes secondaires d'applications (NSA) 66 fournissent des lignes directrices pour l'instruction et la qualification des demandes d'asile en fonction des motifs invoqués et des situations des requérant.e.s rapportés au contexte du pays 67 . Ces NSA tiennent compte de la loi, des ordonnances et directives d'application, de la jurisprudence nationale et internationale, de la doctrine, des informations sur les pays d'origine, ainsi que de l'interprétation institutionnelle du droit pour chaque pays d'origine donné. Ainsi, lorsque les juges du TAF changent leur jurisprudence, le SEM doit, en principe, modifier ses lignes directrices.

À l'inverse, lorsque les «spécialistes asile» et les cadres du SEM veulent changer leur " pratique d'asile», ils et elles peuvent chercher, stratégiquement, à faire évoluer

61. Jérôme DRAHY, Le droit contre l'État?, op. cit.

62. Shauhin TALESH et Jérôme PéLISSE, "How Legal Intermediaries Facilitate or Inhibit Social Change", op. cit.

63. Voir entretiens avec Corinne B., SEM, décembre 2010 et avec Anna F., SEM, mai 2012.

64. Par exemple : entretien avec Stefan M., « spécialiste asile », SEM, octobre 2011.

65. Entretiens avec Carole G., «spécialiste asile ", SEM, mars 2012, et avec Johann D., "spécialiste asile », SEM, juillet 2012.

66. Pierre LASCOUMES, « Normes juridiques et mise en œuvre des politiques publiques », article cité.

67. Jonathan Miaz, «Le Droit et ses médiations. Pratiques d'instruction des demandes d'asile et encadrement institutionnel des décisions ", Politique et Sociétés, 38 (1), 2019, p. 71-98. 
la jurisprudence du TAF avec de nouveaux arguments ou de nouvelles informations sur les pays d'origine 68 :

Parce que ça arrive [qu']on estime qu'il faudrait réorienter la pratique pour ce pays. Et on essaie, nous, de rendre des décisions qui s'écartent un petit peu de la jurisprudence, justement pour peut-être amener le Tribunal administratif fédéral à revoir un peu sa jurisprudence parce qu'on travaille avec des pays où la situation évolue. Et il y a des adaptations qui sont rendues nécessaires par cette évolution 69.

Le SEM peut donc « jouer» stratégiquement de la judiciarisation en tentant des « coups » pour «tester » l'autorité de recours et, in fine, changer le droit via la jurisprudence. Toutefois, en cas de désaccords avec le TAF, maintenir des «pratiques d'asile » contraires à la jurisprudence peut exposer l'administration à des rappels à l'ordre et à des coûts financiers liés aux décisions cassées par le tribunal 70. Les propos d'une "spécialiste asile " expérimentée mettent en évidence le "jeu croisé » entre l'administration, la défense juridique et l'autorité de recours en cas de désaccords sur la «pratique» :

- Jonathan: Et, j'avais entendu que des fois ça arrivait de tester un peu le Tribunal administratif fédéral sur des décisions où, justement, y a un changement dans le contexte par exemple. Ça, ça t'arrive?

— Brigitte $\mathrm{H}$ : Alors, nous ça ne nous arrive plus [de tester le TAF] depuis que j'ai repris la Federführung ${ }^{71}$ [du pays X]. Avant, oui, ça s'est fait, mais c'est pas moi qui m'en occupais. Parce qu'on n'avait pas du tout la même pratique [que le TAF] au niveau des provinces sûres 72 . Nous, on avait des provinces qu'on considérait sûres et le Tribunal pas. Et l'ODM 73 a quand même maintenu sa pratique. Puis, après, il y a eu une décision de principe du TAF sur l'obligation en général, pas seulement pour [le pays $\mathrm{X}$, de se conformer à la pratique du TAF qui est une instance supérieure, pour une raison d'égalité de traitement pour tous les ressortissants. Et puis, après, donc notre pratique a effectivement changé. Donc nos provinces, on a revu, même si on n'était pas d'accord à l'époque. Il y avait une province que, nous, on considérait comme très sûre et puis que le Tribunal considérait comme pas sûre, donc on a continué à renvoyer les gens là-bas, tu vois. Et puis, après, il y a eu cette décision du Tribunal fédéral sur la conformité de la pratique de l'ODM qui doit être par rapport au TAF. Donc on ne s'est plus amusé à faire... on n'a rien testé du tout, puisque c'était une décision de principe. Et puis après, eux, ils ont répondu des décisions sur [le pays X], c'est-à-dire que seules les grandes villes comme [A., B., ou C.], on peut renvoyer des gens avec des conditions très très restrictives, au niveau du réseau social, familial, des possibilités de logement, etc. Et puis, on a tout de suite adapté notre pratique ${ }^{74}$.

68. Damian RosSET, « Le savoir sur les pays d'origine dans les procédures d'asile. Construction et négociation institutionnelle de la réalité», article cité.

69. Entretien avec Sylvain O., « spécialiste asile », SEM, novembre 2011.

70. Entretien avec Andreas S., adjoint juridique, SEM, septembre 2011 et mars 2012.

71. Ici, l'emploi du terme Federführung renvoie au fait d'avoir la responsabilité (souvent à plusieurs) d'une "pratique d'asile " spécifique à un pays d'origine.

72. Le fait de considérer des provinces du pays X comme étant sûres permet au SEM d'y renvoyer des requérant·e.s d'asile dont la demande a été rejetée.

73. L'Office fédéral des migrations (ODM) est l'ancien nom du SEM. Cet office est devenu le Secrétariat d'État aux migrations en 2015.

74. Entretien avec Brigitte H., « spécialiste asile », SEM, juillet 2012. 
Ce jeu stratégique et conflictuel n'implique pas uniquement l'administration, les acteurs de la défense juridique et le Tribunal administratif fédéral. Le Parlement et le Gouvernement sont également impliqués dans cette chaîne d'interdépendances, comme le montre l'exemple de jurisprudence sur l'Érythrée ${ }^{75}$. En 2006, une jurisprudence de la CRA (JICRA 2006/3) avait conduit à l'augmentation du taux de protection (asile et admission provisoire) pour les ressortissant.e.s érythréen·ne.s. En réaction, des politiciens de droite - en particulier le conseiller fédéral Christoph Blocher - ont cherché à durcir la loi sur l'asile (2012) et à forcer l'administration à modifier sa pratique, notamment via de nombreuses questions, motions et interpellations parlementaires. Le SEM a alors essayé de faire changer la jurisprudence en produisant de nouvelles informations sur le pays d'origine. En 2016, suite à un rapport d'une "fact-finding mission" en Érythrée, le SEM a durci sa pratique. Le 30 janvier 2017, les juges du TAF ont finalement modifié leur jurisprudence (D-7898/2015), en considérant que, dans certains cas, les renvois en Érythrée peuvent être considérés comme licites et exigibles.

\section{Conclusion}

En dépit des réticences des autorités suisses à créer une instance de recours indépendante de l'administration susceptible d'influencer la pratique de l'asile et de limiter leur pouvoir discrétionnaire, on observe depuis la mise en place d'une telle juridiction une judiciarisation de la politique suisse d'asile importante tant quantitativement en lien avec le nombre de recours, que qualitativement en termes d'effets de la jurisprudence sur les acteurs de terrain. Cette jurisprudence s'avère contraignante pour les lignes directrices ( pratiques d'asile») de l'administration qu'elle doit revoir en fonction de l'interprétation que le tribunal fait de la situation d'un pays d'origine. Cela la pousse parfois à accorder une protection (asile ou admission provisoire) là où elle estimait qu'il n'y avait pas lieu de reconnaître une persécution ou là où elle considérait que le renvoi était exigible, licite et possible.

Toutefois, dans d'autres cas, le tribunal peut également confirmer les pratiques d'asile de l'administration et leur donner davantage de poids en les inscrivant dans la jurisprudence. De plus, via la phase de consultation qui précède une révision législative et via les personnes qui, au sein du Secrétariat d'État aux migrations, rédigent les projets de loi 76 , l'administration peut aussi essayer de proposer des changements législatifs visant à faire remonter des problèmes pratiques rencontrés sur le terrain et à boucher les failles ouvertes par la jurisprudence. Ce que font également, de leur côté, certain·e.s parlementaires et membres du Gouvernement. Si on peut, à plusieurs égards, parler d'une forme de backlash politique, on ne saurait

\footnotetext{
75. Pour plus de détails, voir: Aurélie MARiotTi et Damian Rosset, «L'analyse-pays et les "précédents factuels" dans la jurisprudence du Tribunal administratif fédéral sur l'Erythrée : entre ombre et lumière", Asyl. Revue Suisse pour la pratique et le droit d'asile, 2, 2020, p. 3-9. Voir aussi le rapport: OSAR, "Analyse des durcissements de la pratique suisse à l'égard de requérant-e-s erythréen-ne-s. Recherche du service juridique», Berne, 13 décembre 2018, téléchargé le 9 juillet 2019: <https://www.osar.ch/assets/news/eritrea/ 181213-recherche-osar-erythree.pdf>.

76. Entretiens avec Aline M. et avec Audrey S., adjointes juridiques, SEM, juin et juillet 2012.
} 
se limiter à cette analyse 77 . En effet, la jurisprudence a aussi établi certaines garanties procédurales et des interprétations plus favorables du droit à l'égard de certains motifs, situations et pays d'origine, ce qui a permis à de nombreuses personnes d'obtenir in fine un statut en Suisse.

Pour les acteurs de la défense juridique, la jurisprudence permet ainsi d'ouvrir des «brèches" (opportunités juridiques) et d'infléchir la pratique des autorités (octroi d'une protection, garanties procédurales). Toutefois, elle fixe aussi toute une série de contraintes et de restrictions dans l'application du droit d'asile qui limitent leur marge de manœuvre et les possibles juridiques pour défendre individuellement et collectivement les migrant-e.s. De plus, en raison de leur manque de ressources et des contraintes liées au langage du droit, les bureaux de consultation juridique sont amenés à sélectionner celles et ceux qu'ils acceptent de défendre en se basant notamment sur les « chances de succès " d'une démarche juridique, soit sur la reproduction partielle de certains critères juridico-administratifs.

Ainsi, la judiciarisation de la politique d'asile peut être considérée comme un vecteur de transformation du droit d'asile et de sa pratique, par la marge, dans un sens plus favorable aux requérant.e.s d'asile, mais aussi parfois dans celui voulu par les autorités politiques. En définitive, l'analyse des usages du droit par différents acteurs de la chaîne d'interdépendances de la politique d'asile en Suisse met en évidence comment se poursuit le conflit politique autour de la politique d'asile sur le terrain de la mise en œuvre. Ce faisant, cet article met en évidence la coproduction conflictuelle du droit d'asile par les pratiques et les interactions entre les pratiques de différents intermédiaires du droit.

\section{- L'auteur}

Jonathan Miaz est chercheur au Centre de droit comparé européen et international de l'Université de Lausanne. Ses recherches portent sur la mise en œuvre des politiques migratoires à l'aune des pratiques des administrations, des tribunaux et des mobilisations. Il travaille actuellement sur les usages politiques du droit international dans les cantons suisses. Il a notamment publié :

— « Entre examen individuel et gestion collective : automatisation des décisions dans la procédure d'asile en Suisse? ?, Lien social et Politiques, 83, 2019 ;

— «Le Droit et ses médiations. Pratiques d'instruction des demandes d'asile et encadrement institutionnel des décisions ", Politique et Sociétés, 38 (1), 2019.

77. C'est aussi ce que montre: Thomas M. KECK, «Beyond Backlash: Assessing the Impact of Judicial Decisions on LGBT Rights ", Law \& Society Review, 43 (1), 2009, p. 151-186. 